\title{
Elke Muchlinski
}

\section{Paradigmenwechsel in der monetären Koordination von Zentralbanken?}

\begin{abstract}
„To suppose that there exists some smoothly functioning automatic mechanism of adjustment which preserves equilibrium if only we trust to methods of laissez-faire is a doctrinaire delusion which disregards the lessons of historical experience without having behind it the support of sound theory" (Keynes, C.W., Vol. XXV, 22).
\end{abstract}

\section{Einleitung}

Die zentrale These des folgenden Beitrags lautet, dass die monetäre Koordination von Zentralbanken, d.h. die Politik der Wechselkursstabilisierung im System flexibler Wechselkurse, durch die Einführung des Euro an Bedeutung gewinnen wird. Diese These soll vor dem Hintergrund der Theorieentwicklung und der Währungsgeschichte untersucht werden. Sie steht konträr zur aktuellen Verlautbarung von Zentralbanken und zu Theorien der monetären Koordination, deren Botschaft lautet: „The market knows“.

Demgegenüber geht der vorliegende Ansatz für die Begründung von Wechselkurspolitik im System flexibler Wechselkurse von drei Prämissen aus. (1) Von der Nichtneutralität des Geldes und des nominalen Wechselkurses; (2) Vom Repräsentativen Realismus und dessen Implikation, dass Modelle einen „link to the contemporary world“ (Keynes) aufweisen müssen. (3) Davon, dass ökonomische Wechselwirkungen zwischen Volkswirtschaften eine Gestaltungsaufgabe begründen, und keineswegs eine Restriktion für die Binnenökonomie.

Während in theoretischen Diskussionen über Währungspolitik die Auffassung dominiert, die Binnenökonomie werde durch außenwirtschaftliche ,Zwänge restringiert', wird in dem vorliegenden Ansatz dieser Blickwinkel umgekehrt (Muchlinski 1999, 1998a). Er geht erstens auf Distanz zur Theorie flexibler Wechselkurse, die eine Abschirmungsfunktion der Binnenökonomie von außenwirtschaftlichen Einflüssen konstatiert. Außenwirtschaftliche Ereignisse werden dort als exogene Schocks modelliert, die die binnenökonomische Strategie konterkarieren und durch Wechselkurse flexibel abgefangen 
werden können. Jener insulare Blickwinkel ist ohne Zweifel Ausdruck eines naiven Konstruktivismus. Die ökonomischen Wechselwirkungen entziehen sich im insularen Denkgebäude der kognitiven Bearbeitung. Die Entscheidung für oder gegen ein System flexibler Wechselkurse erfordert demgegenüber einen Kontextbezug (Frankel 1999). Zweitens distanziert sich der vorliegende Beitrag von dominanten Positionen, die mit „Weltmodellen“ die ökonomischen Wechselwirkungen zwischen Volkswirtschaften zu erfassen suchen. Bezogen auf das Themenfeld internationale monetäre Koordination folgt hieraus, dass Währungsbeziehungen nicht auf der Basis eines universellen Modells, beispielsweise des Regime Choice Approachs (Hamada/Kawai 1997) gestaltet werden können.

Ökonometrische Weltmodelle waren in den 80er Jahren ein Versuch, die „spill-over-Effekte“ von Geld- und Fiskalpolitik zwischen Volkswirtschaften zu quantifizieren. Die Wohlfahrtseffekte einer internationalen Koordination hängen von diesen „spill-over-Effekten“ ab (Cooper 1968). Unter flexiblen Wechselkursen können unterschiedliche Geldpolitiken nationaler Zentralbanken die Wirtschaftspolitik eines anderen Landes konterkarieren. So führt das in der Literatur diskutierte Problem der „beggar-thy-neighbour-policy“ in spieltheoretischen Darstellungen zu Kontraeffekten für die inländische Ökonomie. Daraus folgt, dass eine Überwälzung der Kosten einer inländischen Stabilisierungspolitik auf das Ausland das Problem eines „beggar-yourself“ erst hervorbringt. Hieraus wird im allgemeinen die Bereitschaft von Volkswirtschaften zu Koordination deduziert. Aber auch koordinierte Maßnahmen, etwa eine koordinierte Intervention von Zentralbanken im Devisenmarkt zu Stabilisierung von Wechselkursen, induziert „spill-over-Effekte“. Die Quintessenz der Diskussionen lautete, wenn jedes Land das „eigene Haus in Ordnung halte", dann sei es die Weltwirtschaft als Ganzes auch (Feldstein 1988, Kösters 1990). Währungsgeschichtlich und theoriegeschichtlich manifestiert sich in dieser Sichtweise eine "fallacy of composition“ (Keynes), da die Weltwirtschaft und die Weltwährungsbeziehungen nicht als simple Addition der einzelnen Ökonomien interpretiert werden können. Ein für mich interessantes Phänomen ist hierbei, dass aus den ablehnenden Haltungen zur Koordination von Währungsbeziehungen kein Ende der Debatten folgte. Das Thema ist nach wie vor auf der Agenda.

Die Motivation für den vorliegenden Beitrag resultiert aus dem Fehlen einer kohärenten Theorie der monetären Koordination von Wechselkursen im System flexibler Wechselkurse. Im folgenden Teil werde ich zunächst anhand methodologischer Überlegungen die Verwendung von Universalmodellen erläutern. Daran schließt sich die Präzisierung des Ansatzes der „alten“ und „neuen“ tripolaren Konfiguration von Währungen an. Schließlich folgt ein zusammenfassender Überblick über das Ensemble an Theorien zur monetären Koordination in retrospektiver Sicht. 
„The Lucas critique warns us that some parameters may change when policy does. Yet what are we to do about these problems? Be skeptical? Of course. Use several methods and models instead of just one? Certainly.“ Alan Blinder $(1998,8)$

\section{Methodologische Überlegungen}

Die vorliegende Untersuchung basiert auf der Konzeption des Repräsentativen Realismus, wobei ich dem Ansatz von Bryant (1995), Kenen (1995) und Krugman (1994, 1995) folge. Wen oder was repräsentiert der Repräsentative Realismus? In der Sichtweise des Repräsentativen Realismus ist ein Modell der Ausgangspunkt, nicht aber der finale Punkt einer Untersuchung. Ein Modell setzt sich aus der Interpretationen von Variablen und aus Strukturgleichungen zusammen, die zweckmäßige Abstraktionen von der wahrgenommenen Wirklichkeit sind. Modelle liefern deduktive Propositionen, die jedoch keine Wahrheitsinstanzen darstellen. Im Unterschied zu induktiven Argumenten weisen sie eine logische, keine theoretische Gültigkeit auf. Modellbildung impliziert demnach eine für einen bestimmten Forschungszweck gewählte Abstraktion. „Economic is a science of thinking in terms of models joined to the art of choosing models which are relevant to the contemporary world“ (Keynes 1938, C.W., XIV, 296). Der Terminus „contemporary world“ verweist auf Erfahrbarkeit als einem Kriterium der Modellrepräsentation. Der Repräsentative Realismus ist grundverschieden vom naiven Realismus und ebensowenig ist er identisch mit der Realismusposition des Kritischen Rationalismus, wie ihn Popper vertreten hat. Er unterscheidet sich ferner vom „Transcendental Realism“.

Sozialwissenschaften wie der Ökonomie haftet oftmals das Etikett an, primitive Theorie zu sein, da sie nicht vollständig formalisierbar sind. Die Ökonomie ist keine Formalwissenschaft, an die die Forderung nach Rigorosität zu richten ist. ${ }^{2}$ Die mathematischen Beweisen zugeschriebene Unfehlbarkeit führt zu unbefriedigenden Ergebnissen, da die Anwendung von Mathematik an bestimmte Voraussetzungen gebunden ist, die für die Sozialwissenschaften nicht gegeben sind (Duménil/Levy 1997). Morishima radikalisiert die in der ökonomischen Profession auffindbare Selbstkritik, wenn er von einem „formalen Ästhetizismus“ spricht, der für eine Erfahrungswissenschaft inadäquat ist (1991,70). Ungelöst bleiben Probleme gegenüber der eigenen Forschungsumwelt. Wenn die ökonomische Realität als reine Nomologie behandelt wird und ökonomische Kalküle unabhängig von sich verändernden Kontextfaktoren zum gleichen Resultat führen, dann muß die Ökonomie auch über eine invariante und stabile Symbolik verfügen. Die Makroökonomie, so lautet

1 Lawson (1997) ist der Protagonist des „Transcendental Realism“, auch „Critical Realism“ genannt; kritisch hierzu, vgl. Baert (1996), Muchlinski (2001, 1999a).

2 Vgl. die Debatte im Economic Journal vom November 1998. 
mein Einwand, und dies gilt auch für das Themenfeld der monetären Koordination, verfügt gerade nicht über eine invariante und stabile Symbolik oder entsprechende Kontextfaktoren. Die Frage, ob aus diesem Grund die Resultate der Untersuchungen universaler „Weltmodelle“ zur Quantifizierung von Wohlfahrtsgewinnen aus internationaler Koordination negativ waren, soll an dieser Stelle jedoch nicht untersucht werden (Muchlinski 1998a).

Im Rahmen des Repräsentativen Realismus wird Modellbildung als Ausgangspunkt der Analyse und nicht als deren Schlußpunkt betrachtet. Die Konsequenz hieraus ist evident: Modelle der Erfahrungswissenschaft Ökonomie müssen einen Anwendungsbezug aufweisen, oder mit anderen Worten, einen „link to the contemporary world“ (Keynes) haben. Zweifelsohne gilt für die Ökonomie wie auch für andere Sozialwissenschaften: Der multiparadigmatische Zustand ist der Normalzustand der Erfahrungswissenschaft (Schülein 2001). Das beinhaltet, dass Universalmodelle irrelevant sind. ${ }^{3}$ Und insofern muss auch die monetäre Koordination von Zentralbanken in einem Kontextbezuggesehen werden.

„Die Einsicht, dass die Interdependenz der Staaten die Geld- und Währungspolitik zu einem strategischen Problem werden läßt, trägt für sich genommen wenig zur Erkenntnis bei. Sie rechtfertigt vor allem nicht die ausschließlich mathematische, verallgemeinernde Behandlung dieses Themas. Eine Analyse des Einzelfalls und der Konstellation, unter denen Währungspolitik konkret betrieben wird, wird sich, will man zu fundierten Aussagen über Chancen und Hindernisse auf dem Weg zu einer internationalen Kooperation gelangen, wohl nicht umgehen lassen“" (Reszat 1992, 68).

„The problem of maintaining equilibrium in the balance of payments between countries has never been solved, since methods of barter gave away to use the money and bills of exchange“ (Keynes, C.W., Vol. $\mathrm{XXV}, 21)$.

\section{Nichtneutralität des Geldes und des nominalen Wechselkurses}

Die Prämisse der Nichtneutralität des Geldes und des nominalen Wechselkurses ist keine Trivialität. Die Orientierung an den Funktionen des Geldes, als Wertaufbewahrungsmittel (Vermögen und Reservemedium), Recheneinheit (Fakturierung und Währungs-Peg) und internationales Zahlungsmittel (Vehikel-Währungen und Interventionen) liegt den Portfolioentscheidungen der Zentralbanken und der privaten Anleger zugrunde. ${ }^{4}$ Bezogen auf die Emissionen von internationalen Anleihen und dem internationalen Bankgeschäft (Kapitalmarkt) dominiert der US-Dollar, gefolgt mit großer Distanz von DMark und Yen. Eine Spitzenposition nimmt der US-Dollar auch als Reserverwährung ein. Dies gilt ungeachtet der empirisch ermittelten relativen Ver-

3 Vgl den instruktiven Beitrag von d'Autume/Cartelier (1997)

4 Eine Systematik internationaler Geldfunktionen liefert Krugman (1984); zu den aktuellen Portfoliopräferenzen von Schlüsselwährungen, vgl. Sommer (1999). 
minderung der Dominanz des US-Dollars (McCauley 1999). Wird eine Währung als internationales Zahlungsmittel (sog. Vehikel-Währungen) präferiert, dann zeigt sich dies im Devisenhandel und den finanziellen Transfers im Rahmen von Exporten und Importen. Rechnet man den Fakturierungsanteil der Inlandswährung bei den Exporten heraus, dann dominieren der USDollar und die D-Mark (ECU-Institute 1995). Werden dagegen die EWSWährungen zusammengefaßt, dann verringert sich die Distanz zum USDollar. Die Orientierung von privaten und offiziellen Anlegern auf ein zentrales Medium ist ein theoretisch interessantes Phänomen, dessen Erklärung jenseits transaktionstheoretischer Argumente zu finden ist (Herr 1992). ${ }^{5}$ In der Keynesianischen Terminologie wird diejenige Währung präferiert, die die höchste Liquiditätsprämie aufweist (Keynes 1936, 166f.).

Ein prominenter Ansatz, der von der Neutralitätsannahme ausgeht, ist die Neue Klassische Makroökonomie (NCM). Ihre theoretischen Wurzeln liegen in der Sichtweise von Milton Friedman (Monetarismus) und sie weist eine enge Verbindung zur Hypothese Rationaler Erwartungsbildung (REH) auf. Für die Begründung der monetären Koordination ist dies jedoch dysfunktional. Ihre Prämisse lautet, dass Wirtschaftssubjekte keine systematischen Fehler begehen. „While there can be no doubt that agents learn from their past mistakes, the issue of whether the learning process converges to the rational expectations equilibrium is an open one" (Pesaran 1996, 31). Mit der Hypothese Rationaler Erwartungsbildung wird unterstellt, alle Agenten würden das „wahre“ Modell der Ökonomie, das die Wirklichkeit korrekt beschreibt, kennen. „This is almost informationally as demanding as the REH itself" (Pesaran 1996, 32). Es wird weder begründet, warum Akteure als Entscheidungsreferenz ein Universalmodell wählen, noch, worauf sich diese Gleichförmigkeit in einer Welt, die ja annahmegemäß von individuellen Nutzen- und Gewinnmaximierern bewohnt wird, gründet.

Die REH geht von einem representative agent aus, ${ }^{6}$ also von einer Konzeption der Repräsentation, die nicht identisch mit dem in diesem Beitrag skizzierten Repräsentativen Realismus ist. Diese Methodik betrachtet Modelle als den finalen Punkt der Analyse. Ferner manifestiert sich in der Gleichsetzung von Miniatur- und Marktsystem eine weitere "fallacy of composition“. Auf das Problem, dass mit der intendierten Mikrofundierung der Marktbeziehungen ein logisches Problem entstanden ist, verweist Leeson (1998). Bezogen auf das Themenfeld monetäre Koordination bedeutet dies, dass sie nicht über einen atomistischen Theorieentwurf begründbar ist. $\mathrm{Zu}$ beachten ist, dass Veränderungen von Kontextfaktoren sich nicht in identischen individuellen Veränderungen zeigen. Ferner steht die Annahme der Homogenität von Er-

5 Portfolioänderungen aus transaktionstheoretischer Sicht untersucht Hartmann (1999).

6 Weiterführend die Auseinandersetzung in Hoover (1998), Muchlinski (1999). 
wartungen und Informationen über die Preisentwicklung im Widerspruch zur dezentral organisierten Ökonomie und Weltwirtschaft. Schotter resümiert

„take the theory of rational expectations which claims that people functioning in a macroeconomy make their predictions of economic variables using the same economic model. While this model of expectations formation is the ultimate from the standpoint of economic theory, one can not truly believe it makes sense. (...) While earlier adaptive expectations models were generally perceived as being sensible but not logical, rational expectations models are perceived as logical but making very little sense“ (1996 205).

Im Unterschied zum Erklärungsansatz von Keynes, der herausgearbeitet hat, dass die Erwartungsbildung der Marktteilnehmer aufeinander bezogen ist, findet im Ansatz der REH die Erwartungsbildung ohne die der anderen Teilnehmer - außerhalb von Raum- und Zeitkoordinaten - statt (Muchlinski 1996). Die zentrale Prämisse von NCM und REH lautet: „money does not matter“. Diese Prämisse ist jedoch für die Zentralbankpolitik und die internationale monetäre Koordination dysfunktional (Ball/Croushore 1998, Blinder 1998, Friedman, B. 1996, Hahn 1982, Muchlinski 2000).

„A good methodology for prospective work requires, however, that before speaking about the future I give at least a brief look at the past“" (Malinvaud 1991).

\section{Tripolarität von Währungsbeziehungen}

Die tripolare Konfiguration von Währungen impliziert eine Hierarchie von drei hinsichtlich ihrer Geldfunktionen unterschiedlichen Währungen, die den Kern des internationalen Währungssystems bildet. Dabei unterscheide ich zwischen der „alten“ tripolaren Konfiguration, bestehend aus US-Dollar, DMark und Yen und einer „neuen“ tripolaren Konfiguration, bestehend aus US-Dollar, Euro und Yen. Diese Differenzierung ist bedeutsam, da der Euro, als nicht-nationales Geld von einer nicht-nationalen Zentralbank emittiert, von Anbeginn in einer ganz anderen Weise in Konkurrenz zum US-Dollar steht als die D-Mark. Die Problematik der Emission eines nicht-nationalen Geldes in Euroland resultiert auch aus den institutionellen Regelungen der Europäischen Zentralbank, des Systems Europäischer Zentralbanken und des Prozesses der Beschlußfassung. Diese Aspekte sind Gegenstand aktueller Kontroversen (vgl. Buiter-Issing-Kontroverse 1999).

Mit der tripolaren Konfiguration von Währungen wird kein normativer Ansatz formuliert, sondern ein theoretischer Ansatzpunkt gewählt. Empirische Evidenz über die Signifikanz tripolarer Währungsbeziehungen präsentierten die Bank of International Settlement in ihren aktuellen Jahrensberichten und der Internationale Währungsfond, um nur einige Studien zu nennen. Theoretische Analysen zur „tripolaren Welt“ lieferten in den 80iger Jahren vor allem 
Canzeroni (1982), und Krugman (1984). ${ }^{7}$ Die Konfiguration der Tripolarität bildet die Währungshierarchie indirekt $a b$, indem sie auf den Verweisungszusammenhang von drei, die Geldfunktionen repräsentierenden Währungen und die Nichtneutralität des nominalen Wechselkurses fokussiert. Die Konfiguration der Tripolarität ist jedoch keine stabile Konstruktion.

In seiner Studie über das Wechselkurs- und Währungsarrangement der Zwischenkriegszeit verglich Eichengreen diese mit der Phase nach dem Ende des II. Weltkriegs. Ein zentrales Merkmal für die erste sei das Fehlen von währungspolitischer Koordination gewesen, wofür er zwei zentrale Gründe nennt: (1) Die Zentralbanken waren institutionell abhängig. (2) Die Staaten beschränkten sich darauf, untereinander unzureichende ökonomische Erklärungen über die in vielen Ländern vorherrschende Inflation und die hohe Arbeitslosigkeit auszutauschen. Außer einem zaghaften Versuch eines „Tripartite Agreement“ im Jahre 1936 gab es keine Koordination (Eichengreen 1995). England und die USA traten in eine Phase einer gemeinsamen Hegemonialposition ein, während Frankreich sich dieser als dritte Partei nicht anschliessen wollte (vgl. auch Keynes 1933, C.W. XXI).

Während des Pfund Sterling Standards war es das Pfund, das die Reservefunktion übernahm. Die Bank von England dominierte die Diskontpolitik über einen Zeitraum von fünfzig Jahren und ihre Aktiva- und Passivapositionen dieser Zeit verdeutlichen, dass sie die Funktion eines Weltbankiers ausübte, wobei sie die Kapitalbewegungen über die Variation des Zinssatzes regulierte. Das Sterling-System war ein „two-tier system of banking and money supply“. Als Weltbankier induzierte England kurzfristige Kapitalimporte und langfristige Kapitalexporte und emittierte die Weltliquidität in der eigenen Währung (Tavlas/Ozeki 1992, 19ff.). Die Erosion dieser hegemomialen Position begann während der Inflation im Ersten Weltkrieg und wurde mit der Entscheidung zur Rückkehr zur Goldparität der Vorkriegszeit im Jahre 1925 zementiert (Keynes 1925), womit das Pfund Sterling sich in einer Position der Überbewertung befand. Für die USA bildete die Zwischenkriegszeit demgegenüber den Ausgangspunkt für die mit dem Bretton Woods Arrangement im Jahre 1944 beschlossene Etablierung der Funktion des US-Dollars als Schlüsselwährung (Mundell 1995, McKinnon 1993, Spahn 1998). Allerdings ist festzuhalten, dass die Leitwährungsfunktion nicht auf dieser institutionellen Grundlage beruht und die Währungsgeschichte keinen einzigen Fall kennt, in dem per Gesetz eine Leitwährung geschaffen wurde. Dies könnte bezogen auf den Euro Bedenken provozieren.

7 Für Beiträge aus den 90iger Jahren sei exemplarisch auf Currie/Levine (1993), McCauley (1997), McKibbon (1997), Weber (1996) verwiesen. 


\section{Tripolare Konfiguration: US-Dollar, D-Mark, Yen}

Die USA fungierten von 1945 bis 1980 als Weltbankier, während der Franc im Hintergrund und der Yen in einer ambivalenter Positionierung blieb. Die „alte tripolare Währungskonfiguration“ aus US-Dollar, D-Mark und Yen manifestiert sich im Kontext des Plaza-Agreements 1985 und seiner Vorgeschichte. Mit diesem Agreement wird ein entscheidender Themenwechsel auf den internationalen Gipfeltreffen eingeleitet, der auf der Ebene der akademischen Diskurse eine bis in die Gegenwart reichende Kontroverse über die Ausgestaltung oder Adäquatheit von Wechselkurssystemen provozierte.

Verursacht durch die fragile Dominanz des US-Dollars avancierte das Ziel der Stabilisierung von Wechselkursen zum wichtigsten Thema der wirtschaftspolitischen Agenda. Feldstein resümiert die Bedeutung des Plaza Agreements aus der Sicht der U.S.A:

„Faced with reality that the dollar had been declining for more than six month, Treasury Secretary James Baker abandoned the previous Treasury position that the strong dollar was a measure for foreign investors' approval of the economic policies of the United States. He acknowledged publicly that the high value of the dollar was a serious problem for American industry and, most surprisingly of all, he agreed to participate in coordinated exchange market intervention aimed at lowering the dollar's value“ $(1988,5)$.

Ein kaum greifbares Ergebnis des Plaza-Agreements von September 1985 war die Abkehr von der Politik des „hand-off“ und „benign neglect“ der USA. Die exzessiven Wechselkursbewegungen des US-Dollars liessen die USÖkonomie nicht unverschont (Muchlinski 2000). Brayton erläutert, dass die USA damals ein Bewußtsein dafür entwickelten, von den Wechselkursbewegungen des US-Dollars abhängig zu sein (1997), wobei es ein zentrales Motiv der USA war, die Überbewertung des Dollars zu verringern und eine Aufwertung des Yen zu induzieren. Frankel (1984) betont demgegenüber, die offizielle Version der USA ließe sich so interpretieren, dass sie an einer Neuformierung der Währungsbeziehungen und damit an einer Inthronisierung der japanischen Währung als Schlüsselwährung interessiert waren. Aber die Periode der Aufwertung endete bereits 1986 (Iwami 1993, McKinnon/Ohno 1997). Der intendierte Bilateralismus zwischen Japan und den USA, wie er durch das Yen-US-Dollar-Committee von 1983 dokumentiert wurde, ${ }^{8}$ bildete letztlich den Auftakt für eine monetäre Koordination zur Stabilisierung der Wechselkurse zwischen der Japanischen Zentralbank und der Bundesbank. Funabashi präzisiert:

„Despite US aggressive bilateralism, which played off West Germany against Japan, the West Germans and the Japanese did attempt, albeit sporadically, to explore mutually defensive stance vis-à-vis the US. During the rapid decline of the dollar in April 1986, before the Tokyo Summit, the Bundesbank and the Bank of Japan intervened in the currency market hand in hand" $(1988,172)$.

Funabashi urteilt, dass es Japans Motiv war, diesen „US aggressive bilateria-

8 Vgl. zum Text des Kommuniqués Funabashi $(1988,160)$. 
lism" in bilaterale Vereinbarungen mit der G-5 und später der G-7 zu transformieren (Funabashi 1998). Die Skizze der tripolaren Währungskonstellation darf nicht über die unterschiedlichen Interessen dieser Länder hinweg täuschen. Ebenso sollte erwähnt werden, dass England und Frankreich während dieser Phase zwar weniger in Aktion traten, dennoch müssen sie in ihrer Rolle als marginale Spieler bei der Gesamtbewertung von Vereinbarungen und Vorschlägen mitberücksichtigt werden.

Mit dem Louvre Accord vom 22. Februar 1987 verdeutlichten die G-7Staaten das Ziel, die Wechselkurse zu stabilisieren: „In current circumstances, therefore, they agreed to cooperate closely to foster stability of exchange rates around the current levels... Neither West Germany nor Great Britain was inclined to allow the French to declare the acceptance of a target zone from the balcony of the Louvre Palace" (Funabashi 1988, 182). ${ }^{9}$ So brachten England und Deutschland ihre Einwände gegen Zielzonen zum Ausdruck. Vorsicht schien darüberhinaus hinsichtlich der exakten Definition von Bandbreiten geboten. Baker (USA) warf ein: „....don't let us be too precise“. Und Pöhl schlug vor: „Let's leave everything open and wait and see how the markets responds". Man einigte sich letztlich auf zwei spezifische Punkte: ausgehend von Relationen von 1,8250 DM/1\$ und 153,50 Yen/1 \$ „plus or minus 2,5\% was determined as a first line of defense for mutual intervention on a voluntary basis, while at $5 \%$ consultation on policy adjustment was to be obligatory; between these limits of 2,5\% to 5\%, intervention efforts were expected to intensify" (Funabashi 1988, 186). Bis zum G-5 Treffen im April 1987 wurden diese Interventionsindikatoren strikt vertraulich behandelt; sie überlebten das Louvre Abkommen nur wenige Wochen. ${ }^{10}$

Das Phänomen des „relative decline of the Dollar“ (McCauley 1997) forcierte in der Folge die Diskussion über Wechselkursstabilisierung. Gründe für diese „ups \& downs“ des US-Dollars waren und sind Umschichtungen der Währungsreserven in den Portfolios der Zentralbanken und extreme Schwankungen des Dollarwechselkurses (DalBosco 1998). Die Überbewertung des US-Dollars und die expansive Geldpolitik führten zur Inflation in den USA und spürbarem Vertrauensverlust in den Dollar als Wertaufbewahrungsmedium. Nicht die Aufgabe der Goldbindung des US-Dollars mit dem Smithonian Agreement 1971, sondern der Verlust der Liquiditätsprämie löste erneute Währungskrisen aus. ${ }^{11}$

9 Im Louvre-Accord wird die Vereinbarung über key variables getroffen, an die sich die multilaterale Überwachung (surveillance) und die koordinierenden Maßnahmen ausrichten sollten. Dazu gehörte Wachstum, Inflation, Zahlungsbilanzposition, Geldpolitik und die Performance der Wechselkurse (Funabashi 1988, 181). Die Wechselkursanpassungen, die im Plaza Agreement vereinbart worden waren, sollten fortgeführt werden.

10 Die engen Bandbreiten von Deutschland, England und Japan waren angesichts des Handelsbilanzdefizits der USA nicht haltbar (Williamson/Miller 1987, 67).

11 Mundell verweist auf das Problem der Konzeption des Bretton Woods Systems. „Gold 
Deutlich wird hier, dass die Wahrnehmungen über Marktbeziehungen erheblich divergieren können und somit die Annahme eines Rekurses auf eine universelle Wahrnehmungskonzeption sinnlos ist. Die kognitiven Dissonanzen über die Marktbewertungen von Wechselkursen reichen bis in die Gegenwart hinein. Dennoch so, urteilt Mundell (1996), war diese Diskussion ein qualitativer Fortschritt verglichen mit der Gestaltungsunwilligkeit beim Abschluß des Smithonian Agreement vom August 1971.

Der Konsens über die Bewertungen „des Marktes“ fehlte. Keynes hatte für die Analyse von Marktbewertungen den Begriff „average opinion“ verwendet. „We have reached the (...) degree where we devote our intelligences to anticipating what average opinion expects the average opinion to be“ $(1936,156)$. Diese Referenz bezeichnet Keynes als „conventional judgment“. Damit wird die Unsicherheit nicht eliminiert, aber in einen Wissenskontext (,body of knowledge") transformiert (Muchlinski 1998b). Keynes' Beschreibung des Verhaltens der Akteure im Kontext von Unsicherheit - „we are assuming, in effect, that the existing market valuation, however arrived at, is uniquely correct in relation to our existing knowledge $(. . .)^{\text {“ }}(1936,152)$ ist analog der beispielsweise dem Louvre Accord zugrundeliegenden Unsicherheit über eine Währungskoordination. Wie ich bereits ausführte, ist Wechselkurspolitik im System flexibler Wechselkurse und damit Währungskoordination in der Tat nur als kontextbezogenes Handeln oder Gestalten realisierbar.

Den Kontextbezug stellt Keynes insbesondere in seinen Überlegungen über die Investitionsentscheidungen unter Unsicherheit ins Zentrum. Der potentielle Investor wird als Akteur charakterisiert, der versucht, den „battle of wits“ zu gewinnen: „To beat the gun, as the Americans so well express it, to outwit the crowd, and to pass the bad, or depreciating, half-crown to the other fellow $(1936,155)$. Nun sollen die historischen Ereignisse nicht im einzelnen nachvollzogen werden. Auf der Theorieebene erodierte die normalwissenschaftliche Forschung (im Sinn von Thomas Kuhn) eines dem dualen Denkgebäude verhafteten Konzeptes von festen versus flexiblen Wechselkursen. Die Frage, von welchen Faktoren die volatilen Bewegungen der Wechselkurse ausgelöst werden, führte zu der Erkenntnis, dass sie in den wenigsten Fällen sogenannten Fundamentalfaktoren folgen (De Grauwe 1994). Die Herausbildung der tripolaren Konfiguration war demgegenüber kein politischer Beschluß, sondern resultierte aus dem marktrelevanten Handeln der beteiligten Notenbanken und der Finanzminister. Wichtig für diese Phase der Etablierung einer - wenn auch fragilen - tripolaren Konstellation ist, dass Japan und Deutschland in bezug auf ihre Währungen andere Interessen als die

would again become a good standard of value if and only if it were made stable in terms of commodities“ (1995, 490). „Gold is nobody's liability and nobody can print it“. Schließlich gilt, "whether gold is stable or not depends inter alia on the gold policies of governments“ (Mundell 1995, 489). 
USA hatten, „particularly as a currency for private assets and official reserves - during most of the postwar period. In particular, the two governments limited borrowing by foreign residents in their domestic capital markets in dmark or yen“ (Henning 1994, 317). Kennzeichnend für die „alte tripolare Konfiguration" ist der unterschiedliche Grad der Erfüllung der Geldfunktionen der drei Währungen: Der Yen stand systematisch unter Aufwertungserwartung, ohne die Rolle als Schlüsselwährung einzunehmen und die DM avancierte zur Leitwährung innerhalb des Europäischen Währungssystems, gegen den dokumentierten Widerstand der Bundesbank und die Intention der USA. Währenddessen blieb der US-Dolar ungeachtet seiner Ups \& Downs mit Abstand die zentrale Schlüsselwährung. Im Rahmen der tripolaren Konfiguration, in der er die zentralen Geldfunktionen mit der D-Mark und dem Yen teilte, war der US-Dollar daher mit Abstand die bevorzugte Währung oder, in Keynesianischer Terminologie, die Währung mit der höchsten Liquiditätsprämie. $^{12}$

Hervorzuheben ist, dass sich unter dem Regime der ,alten tripolaren Währungskonfiguration" die von der Bank of International Settlements (BIS) in ihren aktuellen Jahresberichten bezeichneten Ungleichgewichte zwischen den Währungsräumen US-Dollar, D-Mark und Yen zementierten (2000). Die Auswirkungen hiervon, beispielsweise auf die Länder Asiens, sind evident. Zwar könne trotz eines steigenden Leistungsbilanzdefizits der USA von einem Vertrauen in den US-Dollar seitens privater internationaler Vermögensanleger ausgegangen werden, aber die Kapitalimporte hingen signifikant von der künftigen Zinspolitik der Federal Reserve Board ab (BIS 1999, 117). Diese Situation globaler Ungleichgewichte zwischen den USA einerseits (Leistungsbilanzdefizit) und dem DM-Währungsraum und Japan andererseits (Leistungsbilanzüberschuß) wird in der Literatur als überwiegend fragil interpretiert (Mundell 1995, IMF-Annual Report 1999). Die BIS weist in ihrem Schlußkapitel auf den „state of confidence“ hin, der einer weiteren Auslandsverschuldung der USA eine Grenze setzen könnte, die eine um so restriktivere Geldpolitik verlangen würde, je höher die Inflationsgefahren in den USA wären.

„Ein allgemeiner Zinsanstieg, ob von den USA oder von Europa ausgehend, könnte überdies einen weiteren Abzug von Mitteln aus den aufstrebenden Volkswirtschaften bewirken (...). Die Folgen einer solchen Neubewertung lassen sich kaum abschätzen, wenn man bedenkt, dass sich die internationalen Bankeinlagen mit über $700 \mathrm{Mrd}$. \$ im vergangenen Jahr fast vervierfacht haben und dass internationale Wertpapieremissionen kürzlich historische Höchststände erreichten“ (BIS 1999, 183-4).

Eine Studie der Deutschen Bank (2000) kommt zu dem Ergebnis, dass sich die USA als „buyer of last resort“ zwar als stabilisierender Faktor für die Weltwirtschaft verhält, aber dass ein zu hohes Leistungsbilanzdefizit zu einem Vertrauensverlust in den US-Dollar führen könnte. Krugman resümiert die 
vergangene Dekade mit Blick auf Amerikas Führungsrolle in der Weltwirtschaft folgendermaßen:

„Several good years of U.S. growth and two very bad years in Asia have shifted the quantitative picture, but not the qualitative one. The old line surely applies: we were never as bad as people said, and now we aren't as good. Above all, it is very hard to imagine how the kind of supremacy the United States once had - when it outclassed conceivable rival on every dimension you could think of - could ever reemerge. America will not dominate the world economy the way it used to, not because it is doing something wrong, but because many other countries are also doing something right. And that is good news for everyone" (2000).

Mit diesem Zitat möchte ich auf den nächsten Abschnitt überleiten, in dem die Beschreibung der „neuen tripolaren Konfiguration“ und ihre Bedeutung für die monetäre Koordination erfolgen soll.

\section{Tripolare Konfiguration US-Dollar-Euro-Yen}

Die „neue tripolare Währungskonfiguration“ manifestiert sich mit der Einführung des Euros (Muchlinski 1998a, 1999a). Ein zentrales Unterscheidungsmerkmal gegenüber der „alten tripolaren Konfiguration“ ist die Emittierung des Euro als nicht-nationales Geld durch die Europäische Zentralbank und das System der Europäischen Zentralbanken. Die daraus resultierenden Fragen betreffen folgende Aspekte: (1) Umfang und Ausmaß der Vermögensumschichtungen zwischen US-Dollar, Euro und Yen. (2) Die erfolgreiche Etablierung des Euros in den drei genannten Geldfunktionen. Der Unterschied zur D-Mark und zur Bundesbank ist evident. Dornbusch/Favero/Giavazzi (1998) sprechen von einer Gratwanderung, die von der EZB zu leisten ist. Die Etablierung der D-Mark als Schlüsselwährung ist in einen historischen Kontext eingebunden und nicht auf den Euro übertragbar.

Die Währungsgeschichte verdeutlicht, dass formale Arrangements - wie etwa das System von Bretton Woods - nicht hinreichend sind, um eine Schlüsselwährung zu etablieren. Für den Euro lautet die spannende Frage, ob er die ökonomische Performance von Euroland oder zumindest einigen Teilen des Eurolands repräsentiert. Diese Fragen sind nicht irrelevant, wenn es um die Beurteilung der neuen tripolaren Konfiguration von Währungsbeziehungen geht, da in dieser unterschiedliche Zentralbanken die Akteure sind, deren Wechselwirkungen erst noch den sog. „Markttest“ bestehen müssen. Abstrahieren möchte ich hier von den Diskussion über Bipolarität versus Tripolarität. Ich beschränke mich auf eine kurze Anmerkung: Die Reflektionen über die „alte“ und „neue“ Tripolarität sind auch dann noch relevant, wenn z.B. Ilzkowicz (1995) konstatiert, der Euro sei lediglich Teil der amerikanischen Dollar-Zone oder Fratianni et al (1998) verkünden, das internationale Währungssystem sei ein bipolares. Bergsten, der Japan als Juniorpartner charakterisiert, urteilte:

„The euro's rise will convert an international monetary system that has been dominated by the US-Dollar since World War II into a bipolar system“ (1997, 92; vgl. Issing 1998, 24f.). 
Frankel bemerkt zur „popular hypothesis“, das internationale Währungssystem weise in den kommenden Dekaden nur noch drei Währungsblöcke auf, „the dollar and the euro are looking somewhat more credible as bloc anchors than in the past. The yen much less so" $(1999,17){ }^{13}$ Mundell urteilt:

„The introduction of the euro redraws the international monetary landscape. With the euro upon its birth the second most important currency in the world - a tripolar currency world involving the dollar, euro, and yen came into being. The exchange rates among these three islands of stability will become the most important prices in the world economy" $(2000,337)$.

Für die Tripolarität U.S.-Dollar, Euro und Yen ist festzuhalten, dass monetäre Koordination in der aktuellen Debatte kein explizites Ziel ist. Koordinierte Interventionen am Devisenmarkt werden abgelehnt. Jenseits dessen zeigt sich allerdings eine politische Akzeptanz hinsichtlich eines bestimmten Grads an Wechselkursstabilität, ohne dass dieser jedoch expliziert wird. Die intendierte Wechselkursstabilität soll dem Markt überlassen werden und nur im Krisenfalle ex post gestaltet werden, wobei regelmäßig auf die Wirkungslosigkeit des Plaza-Agreements und einigen sich daran anschliessenden selbstverpflichtenden Vereinbarungen der G 3 oder G 7 verwiesen wird, die lediglich eine Einladung zur Spekulation dargestellt hätten. Ein weiteres Beispiel stellt in diesem Zusammenhang der Verweis auf das Europäische Währungssystem (EWS) im Sommer 1992 dar. Eichengreen/Wyplosz argumentieren, dass die bestehenden festen Bandbreiten mit der Interventionsverpflichtung zur Stützung der Paritäten durch die Zentralbanken im EWS realignments vorzunehmen, Wechselkursänderungserwartungen generierten (1993). Die Krise im EWS im Sommer 1992 und im Frühjahr 1993 zeigt darüber hinaus, dass auch ein Verzicht auf realignments die Spekulation beflügeln kann (Obstfeld 1995, 82). In der Literatur werden daher Zielzonen überwiegend nicht als Möglichkeit einer Steuerung der Markterwartungen, sondern als Geiselnahme der Zentralbank durch den Markt und potentiellen Spekulanten interpretiert.

Ein weiterer Grund, weshalb die Debatte („das eigene Haus in Ordnung halten") aus den 80/90iger Jahren nicht auf die tripolare Währungskonfiguration von US-Dollar, Euro und Yen übertragen werden kann, liegt in den theoretischen Implikationen der Erklärungsansätze. Wie eingangs erwähnt, dominierte in den 80iger Jahren die Sichtweise ökonomischer Weltmodelle, die wirtschaftliche Interdependenz im Rahmen der Logik des „menue of choice“ der Phillipskurve erklärten. Ein weiterer Aspekt ist, dass ihre Annahmen durchweg von der Neuen Klassischen Makroökonomie bestimmt werden, inkl. der Hypothese rationaler Erwartungen.

In diesem Zusammenhäng sollen noch einmal die Modelle der Offenen Volkswirtschaft der Federal Reserve Bank (Fed/USA) erwähnt werden, deren

13 Zur Konzeption tripolarer Währungsblöcke und ihren Wirkungen auf die beteiligten Länder, die sich mit ihrer Währung an einen Blocks „peggen“, vgl. Frankel/Wei (1995) und Collignon (2001). 
Bestandteile sowohl die REH, als auch die Hypothese adaptiver Erwartungsbildung sind. Der Rekurs auf ein Universalmodell wird dabei als dysfunktional empfunden (Brayton/Levin/Tryon 1997). Im Diskurs der modernen Zentralbanktheorie wird konstatiert, dass die intendierte Revolution des Paradigmas der NCM nicht stattfand, wie Mishkin (1995) und Krugman (1995) betonen. Eine zu begrüßende Konsequenz ist, dass sich auch der Ansatz der adaptiven Erwartungsbildung im akademischen Diskurs re-etablieren konnte (vgl. Blinder 1998). Dieser Paradigmenwechsel ist auch zwingend für das Themenfeld monetäre Koordination, da die Zentralbanken die wichtigsten Akteure sind.

Festzuhalten bleibt, der Devisenmarkt ist trotz (Frankel 1996) respektive infolge (Krugman 1995) der Revolution der „rational expectations hypothesis“ als imperfekter Markt zu beurteilen. Die Simplifizierung der Theorie der flexiblen Wechselkurse, in der Währungen mit Kartoffeln gleichgesetzt und der Preis von Währungen mit dem Preis von Kartoffeln verglichen wurden, findet keine Akzeptanz mehr in der community of science. Kenen konstatiert: „If goods prices were perfectly flexible, there would be little cause to worry about exchange rate arrangements" $(1994,124)$. Mit Blick auf die Dollarschwankungen in den achtziger Jahren schreibt er:

„If the inhabitants of the market had been endowed with the marvelous attributes displayed by those who populate many economists' models, they would have known that the U.S. budget and trade deficits could not last indefinitely and that the dollar would have to return eventually to something near it 1980 level“ $(1994,124)$.

Wechselkursschwankungen sind in den wenigsten Fällen ihres Auftretens ein Reflex von Gütermarkttransaktionen. Sie sind Ausdruck eines komplizierten Zusammenspiels von Zinsänderungs- und Wechselkursänderungserwartungen. Hierdurch rücken die Zentralbanken als Akteure in den Mittelpunkt (Tobin 1987, 1978). Das Zusammenspiel von Wechselkursänderungserwartungen und internationalen Kapitalbewegungen generiert Wirkungen für die Zinspolitik und Einkommensbildung. Keynes hat dies deutlich gesehen:

„The short term rate of interest is easily controlled by the monetary authority, (...) because it is not difficult to produce a conviction that its policy will not greatly change in the very near future, and also because the possible loss is small compared with the running yield (unless it is approaching vanishing point). But the long-term rate may be more recalcitrant when once it has fallen to a level which, on the basis of past experiences and present expectations of future monetary policy, is considered 'unsafe' (...). For instance, in a country linked to an international gold standard, a rate of interest lower than prevails elsewhere will be viewed with a justifiable lack of confidence (...)“ (1936, 203).

Es ist das Kennzeichen einiger monetärer Ansätze zur Wechselkursbestimmung, mit rigorosen Formulierungen das Problem der Unsicherheit per Annahme eliminiert zu haben. Die Problematik dieser Vorgehensweise pointiert Mayer:

„Users of models should not be dazzled by the technical aspects of the model, but remember that the ultimate goal of a science is not rigor, but a correct and systematic discrimination between valid and invalid propositions" (1996, 202). 
Die Grenzen der Formalisierung in der Ökonomie verdeutlichen sich in der nicht hinterfragten Gleichsetzung von theoretischer Analyse und mathematischer Setzung, nicht aber in der Inkongruenz von Modell und Realität. Die Literatur über internationale Koordination hat einen imposanten Umfang erreicht. ${ }^{14}$ Bryant (1995) vertritt einen Regime Preserving Approach (R.P.A.) der internationalen Koordination, dessen Kern es ist, „kommunikative Gleichgewichte" als Indikatoren der makroökonomischen Performance und der Beurteilung über Währungsbeziehungen zu entwickeln (Muchlinski 1998a, 29f.). Bryants Ansatz versteht sich als theoretischer und methodischer Gegenentwurf zum universellen Optimierungsmodell (P.O.A.). Er geht nicht von der Uniformiertheit und Homogenität der Interessen aus, deren Repräsentation eine Weltwohlfahrtsfunktion sein könne. Er weist diese als „false representations of the political and bureaucratic facts of life" zurück (Bryant 1995a, 419). ${ }^{15}$ Die Stabilisierung und Stabilität von Wechselkursen wird in seinem Ansatz als ein öffentliches Gut betrachtet, vergleichbar der Charakterisierung von Geld als öffentlichem Gut. Nicht nur in diesem Punkt markiert Bryant eine Kontraposition zum P.O.A. und dem Postulat, „the market knows“. Ökonomische Wechselwirkungen zwischen den Volkswirtschaften können nicht mit Rekurs auf die „invisible hand“ intellektuell erfaßt werden. Solch ein Vorgehen gleicht der zu Beginn meiner Ausführungen kritisierten insularen Sichtweise, in der das zu Erklärende ausgeklammert wird.

Angenommen, die Zentralbanken der Tripolarität, Fed (USA), EZB und Bank of Japan könnten sich zu einer expliziten Gestaltung der Wechselkurspolitik im System flexibler Wechselkurse zwischen dem US-Dollar, Euro und Yen entschliessen, dann wäre der Vorschlag von Bryant durchaus anwendbar. Dieser umfasst: (1) Regelmäßige Bestandsaufnahme seitens der G-7-Staaten. (2) Die Erarbeitung eines „baseline outlook“, mit dem ein Austausch über Modelle gewährleistet sein soll. (3) Erörterung der Modellunsicherheit. (4) Reflexionen über „what-if-scenarios“ und Erfahrungsaustausch. (5) Entwickeln von Kriterien, mit denen das analytische Verständnis der markoökonomischen Interaktion verbessert werden soll (Bryant 1995, 437f.).

14 Beiträge zum Thema lieferten Branson et al (1990), Currie/Levine (1993), Canzeroni/Grilli (1996), Cooper, R. (1968), Fischer (1988), Reszat (1986). Die Unsicherheit in der Modellwahl und der Modellinterpretation untersuchen z.B. Frankel/Rockett (1986), Frenkel/Goldstein/Masson (1991, 1988), Goldstein/Masson/Taylor (1992), Goldstein (1994), Ghos/Masson (1991). Hamada/Kawai (1997) liefern einen spieltheoretischen Ansatz. Diese Liste ist exemplarisch. Pionierarbeiten zur ökonomischen Interdependenz im Kontext des Policy Optimizing Approach sind Cooper (1968), Johnson (1954), Meade (1951), Niehans (1984, 1968).

15 Eine Beurteilung aus politikwissenschaftlicher Sicht, liefern Putnam/Bayne (1985). 


\section{Schlußbemerkung}

Eine zentrale Schlußfolgerung meiner Ausführungen ist, dass die Zentralbanken der tripolaren Währungsbeziehung eine implizite Koordination realisieren, da der Verweisungszusammenhang von den Bewertungen des Marktes und ihres emittierten internationalen Geldes bzw. ihrer Währung her sie dazu zwingt. Dies resultiert aus der Prämisse der Nichtneutralität des Geldes und des nominalen Wechselkurses. Die Bewertung durch den Markt impliziert eine Budgetrestriktion für Zentralbanken. Dies gilt auch dann, wenn sich diese nicht in der ausdrücklichen Festlegung von Zielzonen oder Zielwerten der Wechselkurse US-Dollar, Euro und Yen manifestiert. Durch diese implizite monetäre Koordination gestalten die Zentralbanken der tripolaren Währungskonstellation die ökonomischen Wechselwirkungen und bewirken einen „link to the contemporary world“.

\section{Literatur}

Baert, Patrick (1996): Realist Philosophy of the Social Sciences and Economics: A Critique. In: Cambridge Journal of Economics, 20, 513-522..

Ball, Laurence/Croushore, Dean (1998): Expectations and the Effects of Monetary Policy. Working Paper No 98-13, John Hopkins University/Federal Reserve Bank of Philadelphia, June 1998.

Bank of International Settlement (BIS) (2000): 70 Annual Report. 1. April 1999-31 March 2000, Basle, 7. June 2000.

- (1999): 69 Annual Report. 1 April 1998-31 March 1999, Basle, 7. June 1999.

- (1996): Central Bank Survey of Foreign Exchange and Derivatives Market Activity 1995. Published by the Monetary and Economic Department of the BIS in May 1996, Basle.

Bergsten, C. Fred (1997): The US-Dollar and the Euro. In: Foreign Affairs, Volume 76, No. 4, 83-95.

- (1997a): The Impact of the Euro on Exchange Rates and International Policy Cooperation. In: Masson, Paul R./Krueger, Thomas H./Turtelboom, Bart G. (Eds.) (1997): EMU and the International Monetary System. Washington, IMF, 17-48.

Blinder, Alan S. (1998) Central Banking in Theory and Practice. Cambridge/Massachusetts/London.

Branson, William H./Frenkel, Jacob A./Goldstein, Morris (Eds.) (1990): International Policy Coordination and Exchange Rate Fluctuations. University of Chicago Press.

Brayton, Flint/Levin, Andrew/Tryon, Ralph (1997): The Evolution of Macro Models at the Federal Reserve Board. In: Carnegie-Rochester Conference Series on Public Policy, 47, 43-81.

Bretton Woods (1994): Looking to the Future. Commission Report, Staff Review, Background Papers, Washington, D.C.

Bryant, R.C. (1995): International Cooperation in the Making of National Macroeconomic Policies: Where Do We Stand? In: Kenen, P.B. (Ed.) (1995), Understanding Dependence. The Macroeconomics of the Open Economy. Princeton/New Jersey, 392-447.

Buiter, Willem H. (1999): Alice in Euroland. In: Journal of Common Market Studies, Vol. 37, No. 2, pp. 181-209, June 1999.

Canzeroni, M.B. (1982): Exchange Intervention Policy in A Multiple Country World. In: Journal of International Economics 13, 267-289.

Canzeroni, M.B./Ethier, W.J./Grilli, V. (Eds.) (1996): The New Transatlantic Economy. Cambridge.

Collignon, Stefan (2001): In Search of Monetary Stability. From Bretton Woods to Sustainable $E M U$. Hants (forthcoming)

Cooper, Richard (1968): The Economics of Interdependence. New York. 
Currie, David/Levine, Paul (1993): Rules, Reputation, and Macroeconomic Policy Coordination. Cambridge University Press.

Dal Bosco, Elvio (1998): Central Banks' Management of Foreign Exchange Reserves. In: Open Economies Review, 9:S1, 665-683.

D'Autume, A./Cartellier, J. (Eds.) (1997): Is Economics Becoming A Hard Science? Cheltenham/Vermont.

DeGrauwe, Paul (1994): Exchange Rates in Search of Fundamental Variables. Discussion Paper No. 1073. CEPR, London.

Deutsche Bank Research (2000): USA: Bedroht das Leistungsbilanzdefizit die Weltkonjunktur? In: Deutsche Bank Research. Aktuelle Themen, Nr. 171, Frankfurt/Main, Sonderausgabe USA.

Dornbusch, Rudi/Favero, Carlo/Giavazzi, Francesco (1998): Immediate Challenges for the European Central Bank. In: Begg, David/Hagen, Juergen v./Wyplosz, Charles/Zimmermann, Klaus F. (Eds.) (1998) EMU: Prospects and Challenge for the EURO. Oxford/Malden, 15-64.

Duménil, G./Lévy, D. (1997): Should Economics Be A Hard Science? In: D'Autume, A./Cartellier, J. (Eds.) (1997), ibid, 276-304.

Eichengreen, Barry (1995): Central Bank Cooperation and Exchange Rate Commitments: The Classical and the Interwar Goldstandards Compared. In: Financial History Review 2, 99-117.

Eichengreen, Barry/Wypplosz, Charles (1993): The Unstable EMS. Diskussion Paper No 817, Centre for Economic Policy Research, London.

Feldstein, Martin (1988): Distinguished Lecture on Economics in Government: Thinking about International Economic Coordination. In: Journal of Economic Perspectives, Volume 2, Spring 1988, 3-13.

Fischer, Stanley (1988): Macroeconomic Policy. In: Feldstein, Martin (Ed.) (1988) International Economic Cooperation. Chicago, University Press.

Flood, Robert P./Garber, Peter M. (1992): The Linkage between Speculative Attack and Target Zone Models of Exchange Rates: Some Extended Results. In: Krugman, Paul/Miller, Markus (Eds.) (1992) Exchange Rate Targets and Currency Bands. Cambridge/New York, 17-28.

Frankel, Jeffrey A. (1999): No Single Currency System is Right for All Countries Or At All Times. NBER-Working Paper 7338, Cambridge, MA.

- (1996): Recent Exchange-Rate Experience and Proposal for Reform. In: The American Economic Review, Papers and Proceedings, May, 153-158.

- (1984): The Yen/US-Dollar Agreement: Liberalizing Japanese Capital Market. Institute for International Economics, 9, Washington.

Frankel, J.A./Froot, K.A. (Eds.) (1993): Understanding the U.S.US-Dollar in the Eighties: The Expectations of Chartists and Fundamentalists. In: Frankel, J.A. (ed.) (1993) On Exchange Rates. Cambridge/Massachusetts.

Frankel, J.A./Rockett, K. (1986): International Macroeconomic Policy Coordination. When Policy Makers Disagree on the Modell. National Bureau of Economic Research Working Paper No. 2059.

Frankel, J.A./Shang-Jin Wei (1995): Emerging Currency Blocs. In: Genberg, Hans (Ed.) The International Monetary System: Its Institutions and its Future. Springer, Berlin, 111-143.

Fratianni, Michele, et al (1998): From the Gold Standard to a Bipolar Monetary System. In: $O$ pen Economies Review 9:S1, 609-635.

Frenkel. Jacob A./Goldstein, M./Masson, Paul R. (1991): Characteristics of a Successful Exchange Rate System. International Monetary Fund, Washington DC.

- (1988): International Coordination of Economic Policy: Scope, Methods, Effects. National Bureau of Economic Research, Working Paper No. 2670.

Friedman, Benjamin M. (1996): The Rise and Fall of Money Growth Targets as Guidelines for U.S. Monetary Policy. NBER Working Paper No. W5465, Washington, DC.

Funabashi, Yoichi (1998): Tokyo's Depression Diplomacy. In: Foreign Affairs, November/ December, Volume 77, Nr. 6, 26-36.

- (1988): From Plaza to the Louvre. Washington, DC.

Ghos, A.R./Masson, Paul R. (Eds.)(1994): Economic Cooperation in an Uncertain World. Oxford and Cambridge.

- (1991): Model Uncertainty, Learning, and the Gains from Coordination. In: American Economic Review, 81, 465-479. 
Goldstein, M. (1994): Improving Economic Policy Coordination: Evaluating Some New And Some Not-So-New Proposals. In: Kenen, P.B./Papadia, F./Saccomanni, F. (Eds.) (1994): The International Monetary System. Cambridge, 298-324.

Hahn, Frank (1982): Money and Inflation. Oxford

Hamada, Koichi/Kawai, Masahiro (1997): International Economic Policy Coordination: Theory and Policy Implications. In: Fratianni, Michele/Dominick Salvadore/Juergen von Hagen (Eds.) (1997): Macroeconomic Policy in Open Economies. Handbook of Comparative Economic Policies. Westport, 87-147.

Hamada, Koichi (1976): A Strategic Analysis of Monetary Interdependence. In: Journal of Political Economy 83, 677-700.

Hartmann, Phillipp (1998): Currency Composition and Foreign Exchange Markets: The Dollar, the Yen and the Future International Role of the Euro. Cambridge UP.

Henning, C. Randall (1994): Currencies and Politics in the United States, Germany, and Japan. Washington, DC.

Herr, Hansjörg (1992): Geld, Währungswettbewerb und Währungssysteme: theoretische und historische Analyse der internationalen Geldwirtschaft. Frankfurt/Main.

Hoover, K.D. (1998): The New Classical Macroeconomics. In: Davis, J.B./Hands. D.W./Mäki, U. (eds.) (1998): The Handbook of Econmic Methodology. Cheltenham/Northampton, 333-339.

International Monetary Fund (1999): World Economic Outlook. A Survey by the Staff of the $I M F$. Washington, DC.

Ilzkowitz, Fabienne (1995): Recent Developments in the International Use of Currencies: Towards a Tripolar Regime? In: ECU-Institute, International Currency Competition and the Future Role of the Single European Currency. Kluwer Law, 67-95.

Issing, Otmar (1999): The Eurosystem: Transparent and Accountable or 'Willem in Euroland'. In: Journal of Common Market Studies, Vol. 37, No. 3, pp. 503-19, September 1999.

- (1998): Von der D-Mark zum Euro. Auswirkungen auf das Weltwährungssystem und die deutsche Wirtschaft. Tübingen.

Iwami, Toru (1993): The Internationalization of the Yen and Key Currency Questions. University of Tokio, Research Institute for the Japanese Economy, Tokyo.

Johnson, Harry (1954): Optimum Tariffs and Retaliation. In: Review of Economics Studies 21: 142-153.

Kenen, Peter P. (1995): (Ed.) Understanding Interdependence. The Macroeconomics of the Open Economy. Princeton/New Jersey.

-(1994/1990): The Coordination of Macroeconomic Policies. In: Kenen, Peter B. (ed.) (1994): Exchange Rates and the Monetary System. Washington, DC, 107-146.

Keynes, John Maynard (1972): The Collected Writings of John Maynard Keynes. Volume I XXXII, London, Macmillan Press.

- (1936): The General Theory of Employment, Interest and Money. London and Basingstoke.

Kösters, Wim (1990): Wohlfahrtsgewinne durch international koordinierte Wirtschaftspolitik? In: Kantzenbach, E. (1990): Probleme der internationalen Koordination der Wirtschaftspolitik. Schriften des Vereins für Socialpolitik, N.F. 198, 45-70.

Krugman, Paul (2000): Can America Stay on Top? In: Journal of Economic Perspectives, Vol. 14, Number 1, 169-175.

- (1994): Peddling Prosperity. Economic Sense and Nonsense in the Age of Diminised Expectations. New York, London.

- (1995): What Do We Need to Know? In: Understanding Interdependence. The Macroeconomics of the Open Economy. Princeton/New Jersey.

- (1984): The International Role of the US-Dollar: Theory and Prospect. In: Bilson, J.F.O./Marston, R.C. (Eds.) (1984): Exchange Rate Theory and Practice. Chicago/London, 261-278.

Lawson, Tony (1997): Economics and Reality. Routledge.

Leahy, Michael. P. (1997): The US-Dollar as an Official Reserve Currency. In: Open Economies Review, Volume 7, 371-390.

Leeson, Robert (1998): The Ghost I Called I Can't Get Rid of Now: The Keynes-TinbergenFriedman-Phillips Critique of Keynesian Macroeconomics. In: History of Political Economy 30:1, 51-94. 
Mayer, Thomas (1996): The Dark Side of Modeling. In: Medema, S.G./Samuels, W.J. (Eds.) (1996): Foundations of Research in Economics: How Do Economists Do Economics? Aldershot/Hunts, 191-203

McCauley, Robert N. (1999): The Euro and the Dollar, 1998. In: Open Economies Review 10, 91-133.

- (1997): The Euro and The US-Dollar. Essays in International Finance, No. 205, International Finance Section, Princeton University.

McKibbon, W.J. (1997): Empirical Evidence on International Economic Policy Coordination. In: Fratianni, M.U./Salvadore, D./von Hagen, J. (Eds.) (1997): Macroeconomic Policy in Open Economies. Handbook of Comparative Economic Policies, Vol. 5, 148-176.

McKinnon, Roland I./Ohno, Kenichi (1997): US-Dollar and Yen. Resolving Economic Conflict between the United States and Japan. Cambridge/ Massachusetts.

McKinnon, Ronald I. (1993): The Rules of the Game - International Money in Historical Perspective. In: Journal of Economic Literature, 31, -44.

Meade, James (1951): The Theory of International Economic Policy. Vol. 1: The Balance of Payments. London.

Mishkin, Fred S. (1995): The Rational Expectations Revolution: A Review Article of: Preston, J. Miller, Ed. The Rational Expectations Revolution, Readings from the front Line. NBER Working Papers Series, No. 5043, Cambridge/Massachusetts.

Morishima, M. (1991): General Equilibrium Theory in the Twenty-First Century. In: The Economic Journal, Volume I, 69-74.

Muchlinski, Elke (2001): Transcendental Realism oder Transformation der Kategorien? In: Bauer, Leonhard/Hamberger, Klaus et al (Hrsg.) (2001): Gesellschaft Denken. Eine erkenntnistheoretische Standortbestimmung der Sozialwissenschaft. Reihe Politische Philosophie und Ökonomie. Springer Heidelberg

- (2000): Geldmengensteuerung versus Zinssteuerung: Anmerkungen zum „monetaristischen Experiment“ der Federal Reserve Bank (USA). In: Diskussionsbeitraege des Fachbereichs Wirtschaftswissenschaft der Freien Universität Berlin, Nr. 2000/23, Volkswirtschaftliche Reihe. Erscheint in: Heise, Arne (2001): USA-Dekonstruktion eines Modells? Marburg.

- (1999): The Lucas Critique \& Lucasianism - Considering the History of Macroeconomics. Diskussionsbeitraege des Fachbereichs Wirtschaftswissenschaft der Freien Universität Berlin, Nr. 1999/1, Volkswirtschaftliche Reihe.

- (1999a): Transcendental Realism in Economic Theory - ATrack Back to Natural Science? Paper presented to the Third Conference of the European Society of the History of Economic Thought, 26th to 28th February in Valencia/Spain.

- (1999b): Macropolicy within a Tripolar Regime. Diskussionsbeitraege des Fachbereichs Wirtschaftswissenschaft der Freien Universitaet Berlin, Nr. 1999/2, Volkswirtschaftliche Reihe.

- (1998): Euroland, Euro and US-Dollar - Trilogy or Trilemma? Diskussionsbeitraege des Fachbereichs Wirtschaftswissenschaft der Freien Universitaet Berlin, Nr. 1988/42, Volkswirtschaftliche Reihe.

- (1998a): Internationale Koordinierung von Wirtschaftspolitik - Neuorientierungen in der Diskussion. Diskussionsbeitraege aus dem Institut für Volkswirtschaftslehre (520), Universitaet Hohenheim, Nr. 165, Stuttgart.

- (1998b): Beyond Constructivism, Ontological Realism, and Empiricism - Keynes's Economic Theory. In: Diskussionsbeiträge des Fachbereichs Wirtschaftswissenschaft der Freien Universität Berlin, Nr. 1998/51, Volkswirtschaftliche Reihe.

- (1996): Keynes als Philosoph. Berlin.

Mundell, Robert A. (2000): A Reconsideration of the Twentieth Century. In: American Economic Review, Vol. 90, Nr. 3, June 2000, 327-340.

Mundell, Robert, A. (1996): European Monetary Union and the International Monetary System. In: Baldassarri, Mario/Imbriani, Cesare/Salvadore, Dominick (Eds.) (1996): The International System between New Integration and Neo-Protectionism. Houndsmill/Basingstoke, Hampshire/London, 81-128.

- (1995): The International Monetary System: The Missing Factor. In: The Journal of Policy Modeling 17(5), 479-492.

Niehans, Juergen (1984): International Monetary Economics. Johns Hopkins University Press. 
- (1968): Monetary and Fiscal Policies in Open Economies under Fixed Exchange Rates: An Optimization Approach. In: Journal of Political Economy, 76, 893-920.

Obstfeld, Maurice/Rogoff, Kenneth (1995): The Mirage of Fixed Exchange Rates. NBER Working Paper No. 5191, Cambridge/MA.

Pesaran, M.H. (1996): Expectations in Economics. In: Greenaway, D./Bleaney, M./Stewart, I. (Eds.) (1996): A Modern Guide to Economics. London/New York.

Putnam, Robert D./Bayne, Nicholas (1985): Weltwirtschaftsgipfel im Wandel. Bonn

Reszat, Beate (1992): Irrationalitäten und Anomalien als Bestimmungsfaktoren währungspolitischer Entscheidungen. In: Kredit und Kapital 1, 94-109.

- (1986): Wirtschaftliche Interdependenz und internatinale Kooperation. In: Hamburger Jahrbuch für Wirtschafts- und Gesellschaftspolitik, 31. Jahr, 267-291.

Salvadore, Dominick (1998): International Monetary and Financial Arrangements: Present and Future. In: Open Economies Review, 9:S1, 375-415.

- (1998a): The Euro as an International Currency. Paper presented to the Fourth ECSA-World Conference, The European Union and the Euro, Bruxelles, 17-18. September 1998.

Schotter, Andrew (1996): You're Not Making Sense, You're Just Being Logical. In: Medema, S.G./Samuels, W.J. (Eds.) (1996): Foundations of Research in Economics: How Do Economists Do Economics?Aldershot/Hunts, 204-214.

Schülein, Johann August (2001): Schwierigkeiten konnotativer Theorien. In: Bauer, L./Hamberger, K. (Hrsg.) (2001), ibid

Sommer, Albrecht (1999): Die internationale Rolle des Euro. In: Working Papers des Business Institute Berlin an der FHW-Berlin. Hrsg. Bruche, G./Herr, H./Rieger, F. (1999).

Spahn, Heinz-Peter (1998): Leadership and Stability in Key Currency Systems. A simple GameTheoretic Approach. In: Arestis, P./Sawyer, M (Eds.) (1998): The Political Economy of Central Banking. Cheltenham.

Tavlas, George S./Ozeki, Yuzuru (1992): The Internationalization of Currencies: An Appraisal of the Japanes Yen, Washington, International Monetary Fund.

Tobin, James (1987): Agenda for International Coordination of Macroeconomic Policies. In: Volcker, P.A. et al (Eds.) (1987): International Monetary Cooperation: Essays in Honor of Henry C. Wallich, Essays in International Finance, No. 169, 61-69.

- (1978): A Proposal for International Monetary Reform. In: Eastern Economic Journal, Volume 4, Nr. 3-4, 153-159.

Weber, Axel, A. (1996): Foreign Exchange Intervention and International Policy Coordination: Comparing the G3 and EMS experience. In: Canzeroni, M.B./Ethier, W.J./Grilli, V. (Eds.) (1996): The New Transatlantic Economy. Cambridge, 54-113.

Williamson, J./Miller, M. (1987): Targets and Indicators: A Blueprint for the International Coordination of Economic Policy. Washington D.C., Institute for International Economics. 